\title{
Correction to: Third-party signals in equity crowdfunding: the role of prior financing
}

\author{
Simon Kleinert • Christine Volkmann • \\ Marc Grünhagen
}

Published online: 30 November 2018

C) Springer Science+Business Media, LLC, part of Springer Nature 2019

\section{Correction to: Small Bus Econ \\ https://doi.org/10.1007/s11187-018-0125-2}

Unfortunately, the original version of this article was published online with error. The data in Tables 1, 3, 4, 5 and 6 were incorrectly displayed and aligned by the Springer proofreaders/or in the proofread stage of Springer.

The corrected Tables 1, 3, 4, 5 and 6 are shown in the next page.

Further, in Table 6 the authors mistakenly numbered the models "25" and "26," this should be "21" and "22," respectively; including in the footnotes to this table.

The original article has been corrected.

The online version of the original article can be found at https://doi.org/10.1007/s11187-018-0125-2

S. Kleinert $(\bowtie) \cdot$ C. Volkmann · M. Grünhagen Bergische Universität Wuppertal, Gaußstraße 20, 42119 Wuppertal, Germany

e-mail: kleinert@wiwi.uni-wuppertal.de

C. Volkmann

e-mail: volkmann@wiwi.uni-wuppertal.de

M. Grünhagen

e-mail: gruenhagen@wiwi.uni-wuppertal.de 


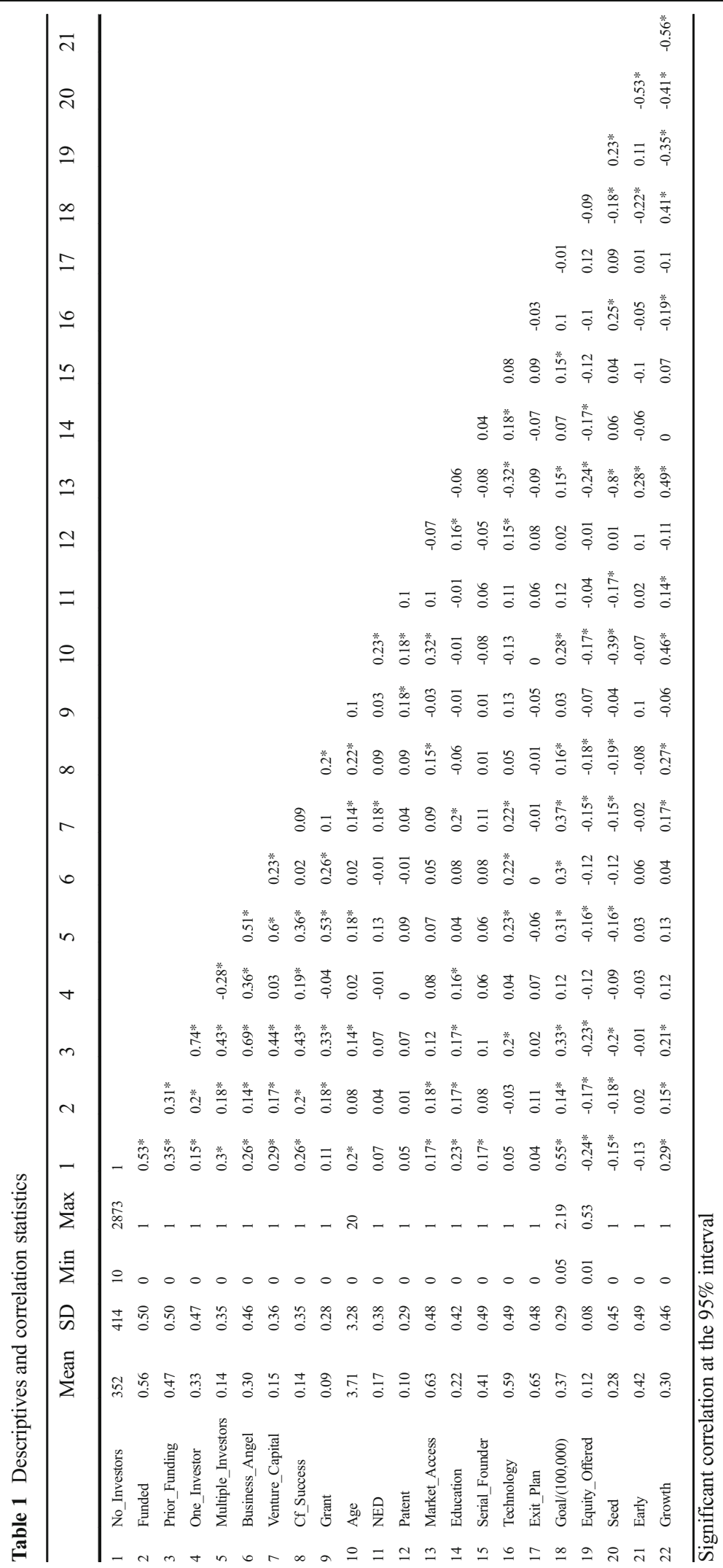


Table 3 Regression analyses for main effects

\begin{tabular}{|c|c|c|c|c|c|c|}
\hline \multirow[t]{2}{*}{ Model } & \multicolumn{3}{|c|}{ DV=No_Investors } & \multicolumn{3}{|l|}{ DV=Funded } \\
\hline & 1 & 2 & 3 & 4 & 5 & 6 \\
\hline Age & $0(0.02)$ & $-0.01(0.02)$ & $-0.01(0.02)$ & $-0.01(0.06)$ & $-0.03(0.06)$ & $-0.01(0.06)$ \\
\hline NED & $0.07(0.15)$ & $0.04(0.14)$ & $0.06(0.14)$ & $0.1(0.49)$ & $0.11(0.5)$ & $0.1(0.49)$ \\
\hline Patent & $0.1(0.2)$ & $0.02(0.2)$ & $0.05(0.2)$ & $-0.14(0.62)$ & $-0.4(0.65)$ & $-0.19(0.62)$ \\
\hline Market_Access & $0.2(0.13)$ & $0.18(0.13)$ & $0.2(0.13)$ & $0.68 *(0.38)$ & $0.77 * *(0.39)$ & $0.69 *(0.38)$ \\
\hline Education & $0.51 * * *(0.13)$ & $0.62 * * *(0.13)$ & $0.56^{* * *}(0.13)$ & $0.83 * *(0.4)$ & $1.04 * * *(0.4)$ & $0.89 * *(0.42)$ \\
\hline Serial_Founder & $0.13(0.11)$ & $0.14(0.11)$ & $0.13(0.11)$ & $0.22(0.32)$ & $0.23(0.32)$ & $0.22(0.32)$ \\
\hline Technology & $-0.19(0.12)$ & $-0.25 * *(0.12)$ & $-0.21 *(0.12)$ & $-0.3(0.33)$ & $-0.34(0.33)$ & $-0.37(0.34)$ \\
\hline Exit_Plan & $0.24 * *(0.12)$ & $0.28 * *(0.12)$ & $0.27 * *(0.12)$ & $0.63 * *(0.32)$ & $0.75 * *(0.33)$ & $0.66^{* *}(0.32)$ \\
\hline $\operatorname{Ln}($ Goal $)$ & $0.51 * * *(0.08)$ & $0.47 * * *(0.08)$ & $0.48 * * *(0.08)$ & $-0.13(0.22)$ & $-0.08(0.23)$ & $-0.17(0.22)$ \\
\hline Equity_Offered & $-1.04(0.74)$ & $-0.92(0.73)$ & $-0.96(0.74)$ & $-1.91(2.26)$ & $-1.81(2.24)$ & $-1.75(2.24)$ \\
\hline Prior_Funding & $0.49 * * *(0.11)$ & & & $1.24 * * *(0.33)$ & & \\
\hline Cf_Success & & $0.52 * * *(0.13)$ & & & $1.14 * *(0.53)$ & \\
\hline Venture_Capital & & $0.37 * *(0.17)$ & & & $0.67(0.55)$ & \\
\hline Business_Angel & & $0.22 *(0.12)$ & & & $0.38(0.37)$ & \\
\hline Grant & & $0.23(0.16)$ & & & $1.56^{* *}(0.74)$ & \\
\hline One_Investor & & & $0.43 * * *(0.11)$ & & & $1.09 * * *(0.34)$ \\
\hline Multiple_Investors & & & $0.7 * * *(0.19)$ & & & $1.79 * * *(0.6)$ \\
\hline Constant & $-0.03(0.77)$ & $0.43(0.8)$ & $0.25(0.77)$ & $0.3(2.04)$ & $-0.17(2.2)$ & $0.73(2.06)$ \\
\hline (Pseudo) $\mathrm{R}^{2}$ & 0.039 & 0.04 & 0.04 & 0.13 & 0.14 & 0.13 \\
\hline Wald chi ${ }^{2}$ & $142 * * *$ & $149 * * *$ & $149 * * *$ & $30 * * *$ & $29 * * *$ & $29 * * *$ \\
\hline
\end{tabular}

Note: Negative binomial regression in models 1-3; logit regression in models 4-6. Robust standard errors in parentheses. $* * * p<0.01$, $* *$ $p<0.05, * p<0.1$ 


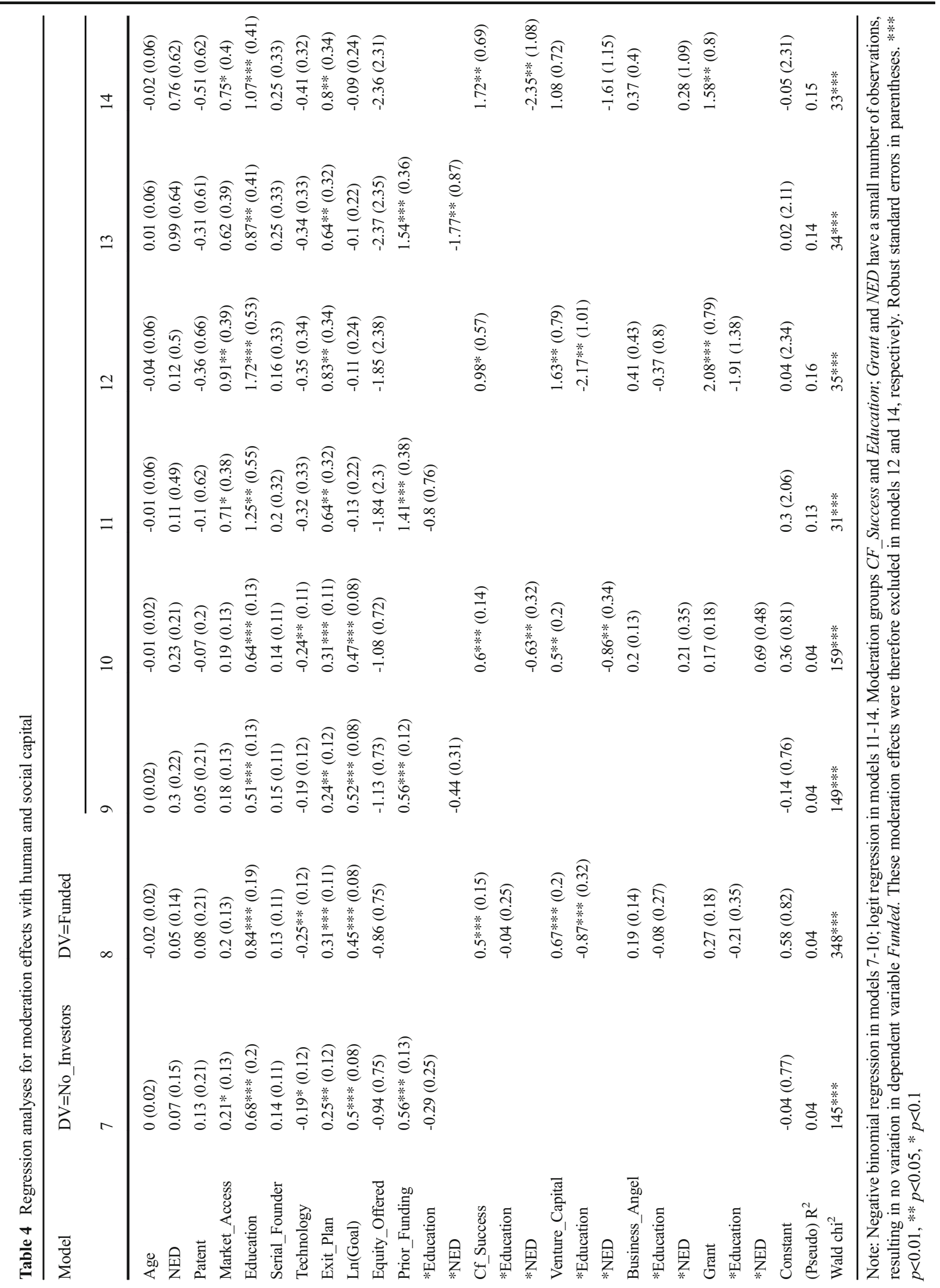


Table 5 Regression analyses for moderation effect with seed

\begin{tabular}{|c|c|c|c|c|c|c|}
\hline \multirow[t]{2}{*}{ Model } & \multirow{2}{*}{$\begin{array}{l}\mathrm{DV}=\mathrm{No} \_ \text {Investors } \\
15\end{array}$} & \multirow{2}{*}{$\begin{array}{l}\text { DV=Funded } \\
16\end{array}$} & \multirow[b]{2}{*}{17} & \multirow[b]{2}{*}{18} & \multirow[b]{2}{*}{19} & \multirow[b]{2}{*}{20} \\
\hline & & & & & & \\
\hline NED & $0.08(0.16)$ & $0.06(0.15)$ & $0.01(0.15)$ & $0.05(0.48)$ & $0.01(0.46)$ & $-0.07(0.49)$ \\
\hline Patent & $0.12(0.22)$ & $0.07(0.21)$ & $-0.08(0.2)$ & $-0.19(0.57)$ & $-0.21(0.56)$ & $-0.37(0.61)$ \\
\hline Education & $0.52 * * *(0.15)$ & $0.51 * * *(0.15)$ & $0.65 * * *(0.15)$ & $0.85^{* *}(0.4)$ & $0.89 * *(0.41)$ & $1.06^{* * *}(0.4)$ \\
\hline Serial_Founder & $0.24 *(0.13)$ & $0.25^{*}(0.13)$ & $0.24 *(0.12)$ & $0.17(0.31)$ & $0.21(0.31)$ & $0.23(0.31)$ \\
\hline Technology & $-0.02(0.14)$ & $0(0.14)$ & $-0.11(0.13)$ & $-0.35(0.33)$ & $-0.31(0.32)$ & $-0.37(0.34)$ \\
\hline Exit_Plan & $0.27 * *(0.14)$ & $0.26^{*}(0.14)$ & $0.31 * *(0.12)$ & $0.64 * *(0.31)$ & $0.62 * *(0.31)$ & $0.74 * *(0.32)$ \\
\hline Equity_Offered & $-0.24(0.79)$ & $-0.06(0.8)$ & $0.23(0.8)$ & $-2.02(2.22)$ & $-1.71(2.22)$ & $-1.5(2.22)$ \\
\hline Seed & $-0.25(0.17)$ & $-0.5 * *(0.2)$ & $-0.52 * * *(0.2)$ & $-0.55(0.38)$ & $-1.16 * *(0.47)$ & $-0.91 * *(0.43)$ \\
\hline Growth & $0.45 * * *(0.16)$ & $0.48 * * *(0.16)$ & $0.36^{* *}(0.15)$ & $0.13(0.38)$ & $0.2(0.37)$ & $0.17(0.39)$ \\
\hline Prior_Funding & $0.66 * * *(0.13)$ & $0.5 * * *(0.15)$ & & $1.14 * * *(0.32)$ & $0.73 * *(0.36)$ & \\
\hline$*$ Seed & & $0.63 * *(0.27)$ & & & $1.7 * *(0.77)$ & \\
\hline Cf_Success & & & $0.52 * * *(0.17)$ & & & $1.03 *(0.54)$ \\
\hline$*$ Seed & & & $0.45(0.4)$ & & & \\
\hline Venture_Capital & & & $0.44 * *(0.21)$ & & & $0.66(0.54)$ \\
\hline * Seed & & & $0.89 * *(0.4)$ & & & \\
\hline Business_Angel & & & $0.33 * *(0.16)$ & & & $-0.02(0.39)$ \\
\hline * Seed & & & $0.53 *(0.3)$ & & & $1.38(0.86)$ \\
\hline Grant & & & $0.12(0.21)$ & & & $1.4 *(0.83)$ \\
\hline$*$ Seed & & & $0.32(0.39)$ & & & $0.32(1.64)$ \\
\hline Constant & $4.94 * * *(0.18)$ & $5.01 * * *(0.18)$ & $5 * * *(0.18)$ & $-0.35(0.48)$ & $-0.25(0.49)$ & $-0.32(0.48)$ \\
\hline (Pseudo) $\mathrm{R}^{2}$ & 0.029 & 0.03 & 0.035 & 0.123 & 0.141 & 0.143 \\
\hline Wald chi ${ }^{2}$ & $92 * * *$ & $102 * * *$ & $153 * * *$ & $30 * * *$ & $35 * * *$ & $32 * * *$ \\
\hline
\end{tabular}

Note: Negative binomial regression in models 15-17; logit regression in models 18-20. Moderation groups Cf_Success and Seed; Venture_Capital and Seed have a small number of observations, resulting in no variation in dependent variable Funded. These moderation effects were therefore excluded in model 20 . Robust standard errors in parentheses. *** $p<0.01, * * p<0.05, * p<0.1$ 
Table 6 Timing of last funding round

\begin{tabular}{lll}
\hline Model & $\begin{array}{l}\text { DV=No_Investors } \\
21\end{array}$ & $\begin{array}{l}\text { DV=Funded } \\
22\end{array}$ \\
\hline Age & $0.01(0.02)$ & $-0.01(0.07)$ \\
NED & $0.09(0.16)$ & $0.05(0.53)$ \\
Patent & $-0.02(0.22)$ & $-0.31(0.67)$ \\
Market_Access & $0.2(0.13)$ & $0.7 *(0.39)$ \\
Education & $0.53 * * *(0.13)$ & $0.8 * *(0.4)$ \\
Serial_Founder & $0.16(0.11)$ & $0.15(0.34)$ \\
Technology & $-0.2(0.12)$ & $-0.25(0.35)$ \\
Exit_Plan & $0.29 * *(0.12)$ & $0.81 * *(0.34)$ \\
Ln(Goal) & $0.5 * * *(0.08)$ & $-0.01(0.22)$ \\
Equity_Offered & $-1.03(0.77)$ & $-2.85(2.51)$ \\
Last_Funding_t0 & $0.66 * *(0.2)$ & $1.42 * *(0.63)$ \\
Last_Funding_t1 & $0.41 * * *(0.13)$ & $0.92 * *(0.4)$ \\
Last_Funding_t2 & $0.45 *(0.23)$ & $1.05(0.69)$ \\
Last_Funding_t $>2$ & $0.28(0.21)$ & $1.93 *(1.13)$ \\
Constant & $-0.02(0.77)$ & $-0.81(2.11)$ \\
(Pseudo) R ${ }^{2}$ & 0.04 & 0.14 \\
Wald chi ${ }^{2}$ & $153 * * *$ & $29 * * *$ \\
\hline Not_Negative & & \\
\hline & &
\end{tabular}

Note: Negative binomial regression in model 21; logit regression in model 22. Robust standard errors in parentheses. $* * * p<0.01$, ** $p<0.05, * p<0.1$

Note: The graphs are based on predicted values of our observations using the estimation models shown in Table 5

Publisher's Note Springer Nature remains neutral with regard to jurisdictional claims in published maps and institutional affiliations. 